\title{
Association between p53 Expression and Amount of Tumor-Infiltrating Lymphocytes in Triple-Negative Breast Cancer
}

\author{
Miseon Lee ${ }^{1 *}$. In Ah Park ${ }^{1 *}$ \\ Sun-Hee Heo ${ }^{1,2}$. Young-Ae Kim ${ }^{1,2}$ \\ Gyungyub Gong ${ }^{1} \cdot$ Hee Jin Lee ${ }^{1}$ \\ ${ }^{1}$ Department of Pathology, Asan Medical Center, \\ University of Ulsan College of Medicine, Seoul; \\ ${ }^{2}$ Asan Center for Cancer Genome Discovery, \\ Asan Institute for Life Sciences, Asan Medical \\ Center, University of Ulsan College of Medicine, \\ Seoul, Korea \\ Received: November 20, 2018 \\ Revised: February 7, 2019 \\ Accepted: February 8, 2019 \\ Corresponding Author \\ Hee Jin Lee, MD, PhD \\ Department of Pathology, University of Ulsan \\ College of Medicine, Asan Medical Center, \\ 88 Olympic-ro 43-gil, Songpa-gu, Seoul 05505, \\ Korea \\ Tel: +82-2-3010-5889 \\ Fax: +82-2-472-7898 \\ E-mail: backlila@gmail.com \\ *Miseon Lee and In Ah Park contributed equally to \\ this article.
}

\begin{abstract}
Background: Most triple-negative breast cancers (TNBCs) have a high histologic grade, are associated with high endoplasmic stress, and possess a high frequency of TP53 mutations. TP53 missense mutations lead to the production of mutant p53 protein and usually show high levels of p53 protein expression. Tumor-infiltrating lymphocytes (TILs) accumulate as part of the anti-tumor immune response and have a strong prognostic and predictive significance in TNBC. We aimed to elucidate the association between p53 expression and the amount of TILs in TNBC. Methods: In 678 TNBC patients, we evaluated TIL levels and expression of endoplasmic stress molecules. Immunohistochemical examination of p53 protein expression was categorized into three groups: no, low, and high expression. Results: No, low, and high p53 expression was identified in $44.1 \%$ $(n=299), 20.1 \%(n=136)$, and $35.8 \%(n=243)$ of patients, respectively. Patients with high p53 expression showed high histologic grade $(p<.001)$, high TIL levels $(p=.009)$, and high expression of endoplasmic reticulum stress-associated molecules ( $p$-elF2a, $p=.013$; XBP1, $p=.007$ ), compared to patients with low $p 53$ expression. There was no significant difference in disease-free $(p=.406)$ or overall survival rates $(p=.444)$ among the three p53 expression groups. Conclusions: High p53 expression is associated with increased expression of endoplasmic reticulum stress molecules and TIL influx.
\end{abstract}

Key Words: p53; Breast neoplasms; Lymphocytes, Tumor-infiltrating
Triple-negative breast cancer (TNBC) is a molecular subtype of breast cancer that lacks expression of estrogen and progesterone receptors and does not show overexpression of human epidermal growth factor 2 . This profile has no targeted therapy or standard biomarkers, and its aggressive phenotype, lower overall survival, and shorter relapse-free intervals than other breast cancer subtypes make its clinical management a challenge. ${ }^{1,2}$ Different biomarkers for TNBC as prognostic or predictive indicators and possible targets for novel therapy have been studied. ${ }^{2,3}$ Insight into the connection between the immune system and breast cancer may improve treatments and outcomes.

We previously demonstrated that TNBC tumors characteristically possess more tumor-infiltrating lymphocytes (TILs) than other breast cancer subtypes and TILs are positively correlated with endoplasmic reticulum stress-associated molecules. ${ }^{4-8}$ The endoplasmic reticulum is responsible for protein folding in cells. ${ }^{9,10}$
In tumor environments, the combination of high cancer cell proliferation rates, nutrient deficiency, and hypoxia lead to accumulation of unfolded or misfolded proteins in the endoplasmic reticulum that induce endoplasmic reticulum stress. Tumor cells activate the unfolded protein response and various downstream endoplasmic reticulum stress signaling pathways to adapt to this environment. ${ }^{9,10}$ Recent studies show that endoplasmic reticulum stress-associated molecules play important roles in tumorigenicity in TNBC.?

The TP53 gene is a tumor suppressor gene that regulates the cell cycle, cell proliferation, DNA repair, cellular senescence, and death by apoptosis. ${ }^{11}$ Cells with somatic TP53 mutations can avoid apoptosis and progress to malignant tumor cells. Tumors with TP53 mutations are highly invasive, poorly differentiated, and have a high histologic grade, showing poor response to chemotherapy. ${ }^{2,12}$ In solid cancers, mutations affecting the protein- 
encoding reading frame, often referred to as null mutations, result in $\mathrm{p} 53$ protein absence. ${ }^{11}$ On the contrary, TP53 missense mutations may lead to the production of a mutant $\mathrm{p} 53$ protein, which has a prolonged half-life relative to the normal isoform that leads to its accumulation in tumor cells and makes it readily detectable by immunohistochemistry. ${ }^{11,13-15}$ Mutant p 53 proteins not only lose the tumor suppressor function of wild-type p53 but also acquire new functions not present in the wild-type protein, termed gain-of-function properties, that promote tumorigenesis. So far, mutant p53 gain-of-function properties have been shown to stimulate tumor cell proliferation, migration, invasion, survival, chemoresistance, cancer metabolism, and tissue architecture disruption. ${ }^{16}$

Both types of mutations (null and missense) have been observed in the same cancer type. ${ }^{11}$ TP53 mutations are seen in 18\%-25\% of primary breast cancers ${ }^{2,17}$ and in approximately $80 \%$ of TN$\mathrm{BCs}$, which is markedly more frequent than in other breast cancer subtypes. ${ }^{2,18,19}$ Mutations in TP53 are predominantly missense mutations, producing mutant $\mathrm{p} 53$ proteins. Furthermore, as the mutant protein in malignant cells is less susceptible to degradation than wild-type $\mathrm{p} 53$, its accumulation establishes the TP53 mutation as an attractive therapeutic target for TNBC..$^{20,21}$

Considering that TNBC is highly correlated with TIL levels, endoplasmic reticulum stress-associated molecules, and TP53 mutation rate, we examined the correlations among TIL levels, endoplasmic reticulum stress-associated molecules, and expression of $\mathrm{p} 53$ in TNBC.

\section{MATERIALS AND METHODS}

\section{Patients and tissue specimens}

A total of 678 TNBC patients who underwent surgery for primary breast cancer between 2004 and 2010 at Asan Medical Center in Seoul, Korea were retrospectively selected. In this group, 470 patients did not present with lymph node metastasis, and they received four cycles of adjuvant anthracycline $\left(60 \mathrm{mg} / \mathrm{m}^{2}\right.$ adriamycin) and cyclophosphamide $\left(600 \mathrm{mg} / \mathrm{m}^{2}\right)$. The remaining 208 patients presented with lymph node metastases and were treated with four cycles of adriamycin, followed by either four cycles of paclitaxel $\left(175 \mathrm{mg} / \mathrm{m}^{2}\right)$ or four cycles of docetaxel $\left(75 \mathrm{mg} / \mathrm{m}^{2}\right)$. In total, 548 patients $(80.8 \%)$ received radiotherapy. The median follow-up period was 78.3 months. Clinicopathologic information and survival data were obtained from medical records and surgical pathology reports. Exemption from informed consent after de-identification of information was approved by the Institutional Review Board of Asan Medical Center (2013-0866).

\section{Histological evaluation}

Two pathologists (H.J.L. and G.G.) reviewed whole sections of the hematoxylin and eosin-stained slides for histologic grade, $\mathrm{pT}$ category, $\mathrm{pN}$ category, and necrosis in the invasive area. Additionally, the levels of stromal TILs were evaluated, using full sections in 10\% increments (defined as the mean percentage of plasma cells and lymphocytes in stoma of invasive carcinoma; if $<10 \%$ area, then 0,1 , or $5 \%$ level criteria were used). ${ }^{22}$

\section{Tissue microarray construction and immunohistochemical evaluation}

Available formalin-fixed paraffin-embedded blocks of 678 cases were arrayed with a tissue-arraying instrument, as previously described. ${ }^{8}$ Tissue microarray sections were stained with an automatic immunohistochemical staining device (Benchmark XT, Ventana Medical Systems, Tucson, AZ, USA). Antibodies to target phospho-eukaryotic initiation factor 2a (p-eIF2a), protein kinase RNA-like endoplasmic reticulum kinase (PERK), X-box binding protein-1 (XBP1), and CD8 were used, and their expressions in the tumor cells were determined, as previously described. ${ }^{8,23}$ An additional antibody to target p 53 (1:1,500, Dako, Glostrup, Denmark) was also used. The level of p 53 expression was ranked on a 4-point intensity scale ( 0 , none; 1 , mild; 2 , moderate; and 3 , intense). The percentage of nuclear expression of the p 53 was also measured. An "immunoreactive score" was generated as the product of the intensity and the percentage of positive cells.

\section{Statistical analysis}

All statistical analyses were performed using SPSS statistical software ver. 18.0 (SPSS, Chicago, IL, USA). The chi-squared test, linear-by-linear association test, Spearman's correlation, Mann-Whitney U test, and log-rank test were used to evaluate the data. All tests were two-sided and statistical significance was set at $\mathrm{p}<.05$.

\section{RESULTS}

\section{Clinicopathologic characteristics of the study population}

All 678 patients were female, and their median age was 47 years at diagnosis (range, 23 to 76 years). Histologic grades 1 and 2 occurred in 160 cases (23.6\%), and grade 3 in 518 cases (76.4\%). There were $299 \mathrm{pT} 1$ tumors $(44.1 \%), 353 \mathrm{pT} 2$ tumors (52.1\%), 25 pT3 tumors (3.7\%), and one pT4 tumor (0.1\%). Most $(n=470)$ of the patients did not have pathologic lymph node metastasis ( $\mathrm{pN} 0,69.3 \%$ ) while 120 tumors $(17.7 \%)$ were 
pN1, 46 tumors (6.8\%) were pN2, and 42 tumors (6.2\%) were $\mathrm{pN} 3$. The tumors were categorized into four groups based on TIL amounts: < 10\% TILs (164 patients, 24.2\%), $\geq 10 \%$; <30\% TILs (154 patients, $22.7 \%$ ); $\geq 30 \%$; and < 60\% TILs (148 patients, 21.8\%), $\geq 60 \%$ TILs (212 patients, 31.3\%). Necrosis in the invasive area was identified in 488 of the patients (72.0\%).

\section{Classifying p53 expression as none, low, or high}

Immunochemical analysis of p 53 expression was scored as follows (Fig. 1): 0, no expression (299 cases, 44.1\%); >0 to 240, low expression (136 cases, 20.1\%); and > 240 to 300, high expression (243 cases, $35.8 \%$ ).

\section{Characteristics of tumors according to p53 expression}

Compared to the low p53 expression group, the high p53 expression group was significantly associated with higher histologic grade $(\mathrm{p}<.001)$, higher TIL levels $(\mathrm{p}=.009)$, increased presence of necrosis in the invasive area $(\mathrm{p}=.010)$, and higher expression of two endoplasmic reticulum stress-associated molecules ( $\mathrm{p}$ eIF2a, $\mathrm{p}=.013$ and XBP1, $\mathrm{p}=.007$ ) (Table 1, Fig. 2). Compared to the no $\mathrm{p} 53$ expression group, the high $\mathrm{p} 53$ expression

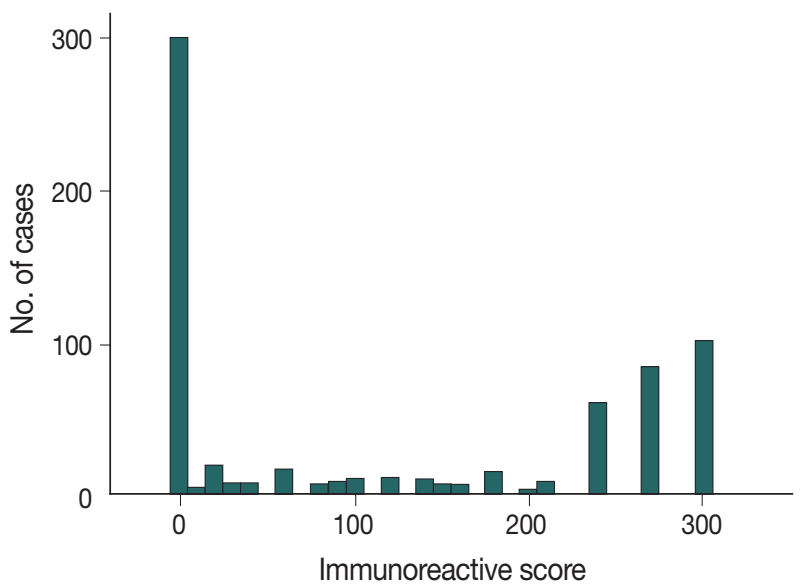

Fig. 1. Distribution of triple-negative breast cancer patients by immunoreactive scores for p53 expression.

Table 1. Comparison of clinicopathologic variables according to p53 expression level

\begin{tabular}{|c|c|c|c|c|c|c|}
\hline \multirow{2}{*}{ Parameter } & \multicolumn{3}{|c|}{ p53 expression level } & \multicolumn{3}{|c|}{$p$-value } \\
\hline & Low (\%) & No (\%) & High (\%) & Low vs no & Low vs high & No vs high \\
\hline Histologic grade & & & & .011 & $<.001$ & .088 \\
\hline 1 and 2 & $48(35.3)$ & $70(23.4)$ & $42(17.3)$ & & & \\
\hline 3 & $88(64.7)$ & $229(76.6)$ & $201(82.7)$ & & & \\
\hline pT category & & & & .103 & .430 & .408 \\
\hline 1 & $70(51.4)$ & $121(40.5)$ & $108(44.5)$ & & & \\
\hline 2 & $61(44.9)$ & $168(56.2)$ & $124(51.0)$ & & & \\
\hline 3 & $5(3.7)$ & $9(3.0)$ & $11(4.5)$ & & & \\
\hline 4 & 0 & $1(0.3)$ & 0 & & & \\
\hline pN category & & & & .510 & .636 & .161 \\
\hline 0 & 95 (69.9) & $199(66.6)$ & $176(72.4)$ & & & \\
\hline $1-3$ & $41(30.1)$ & $100(33.4)$ & $67(27.6)$ & & & \\
\hline TIL & & & & .524 & .009 & .032 \\
\hline$<10 \%$ & $39(28.7)$ & $72(24.1)$ & $53(21.8)$ & & & \\
\hline$\geq 10 \%$ and $<30 \%$ & $33(24.3)$ & $77(25.8)$ & $44(18.1)$ & & & \\
\hline$\geq 30 \%$ and $<60 \%$ & $33(24.3)$ & $65(21.7)$ & $50(20.6)$ & & & \\
\hline$\geq 60 \%$ & $31(22.7)$ & $85(28.4)$ & $96(39.5)$ & & & \\
\hline Necrosis in the invasive area & & & & .579 & .010 & .015 \\
\hline Negative & $46(33.8)$ & $92(30.8)$ & $52(21.4)$ & & & \\
\hline Positive & 90 (66.2) & 207 (69.2) & 191 (78.6) & & & \\
\hline p-elF2a & & & & .174 & .013 & .191 \\
\hline Low & 79 (59.0) & $151(51.4)$ & $108(45.4)$ & & & \\
\hline High & 55 (41.0) & $143(48.6)$ & $130(54.6)$ & & & \\
\hline PERK & & & & .300 & .740 & .487 \\
\hline Low & 77 (57.0) & $153(51.5)$ & $132(54.8)$ & & & \\
\hline High & $58(43.0)$ & $144(48.5)$ & $109(45.2)$ & & & \\
\hline XBP1 & & & & .137 & .007 & .088 \\
\hline Low & $86(64.7)$ & 167 (56.6) & $119(50.0)$ & & & \\
\hline High & 47 (35.3) & $128(43.4)$ & $119(50.0)$ & & & \\
\hline
\end{tabular}

TIL, tumor-infiltrating lymphocyte. 

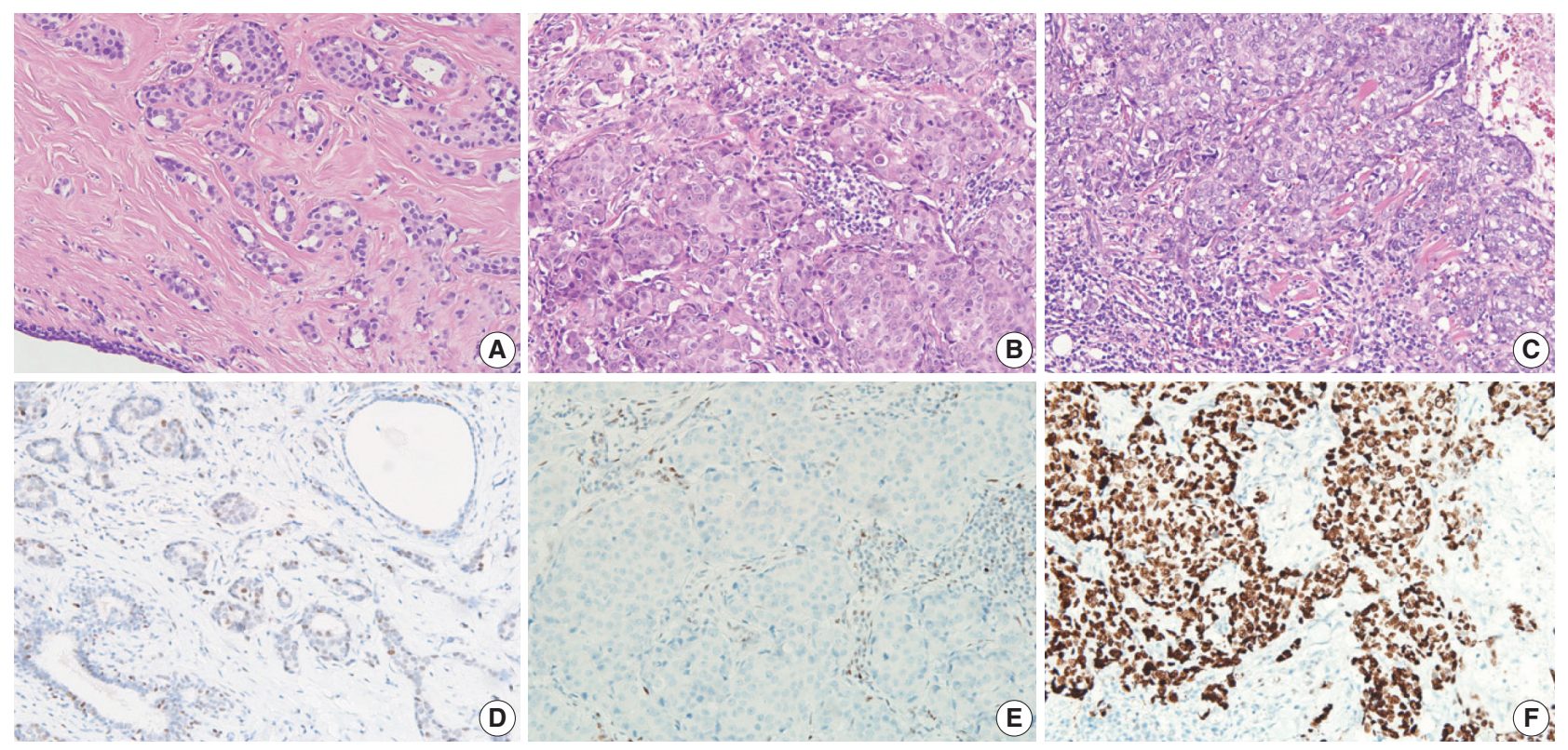

Fig. 2. Histologic features and p53 expression in triple-negative breast cancer tumors. A tumor with low histologic grade shows sparse tumor-infiltrating lymphocytes (TILs) and low p53 expression (A, D). Tumors with high histologic grade show abundant TILs and no p53 expression (B, E) or high p53 expression (C, F).
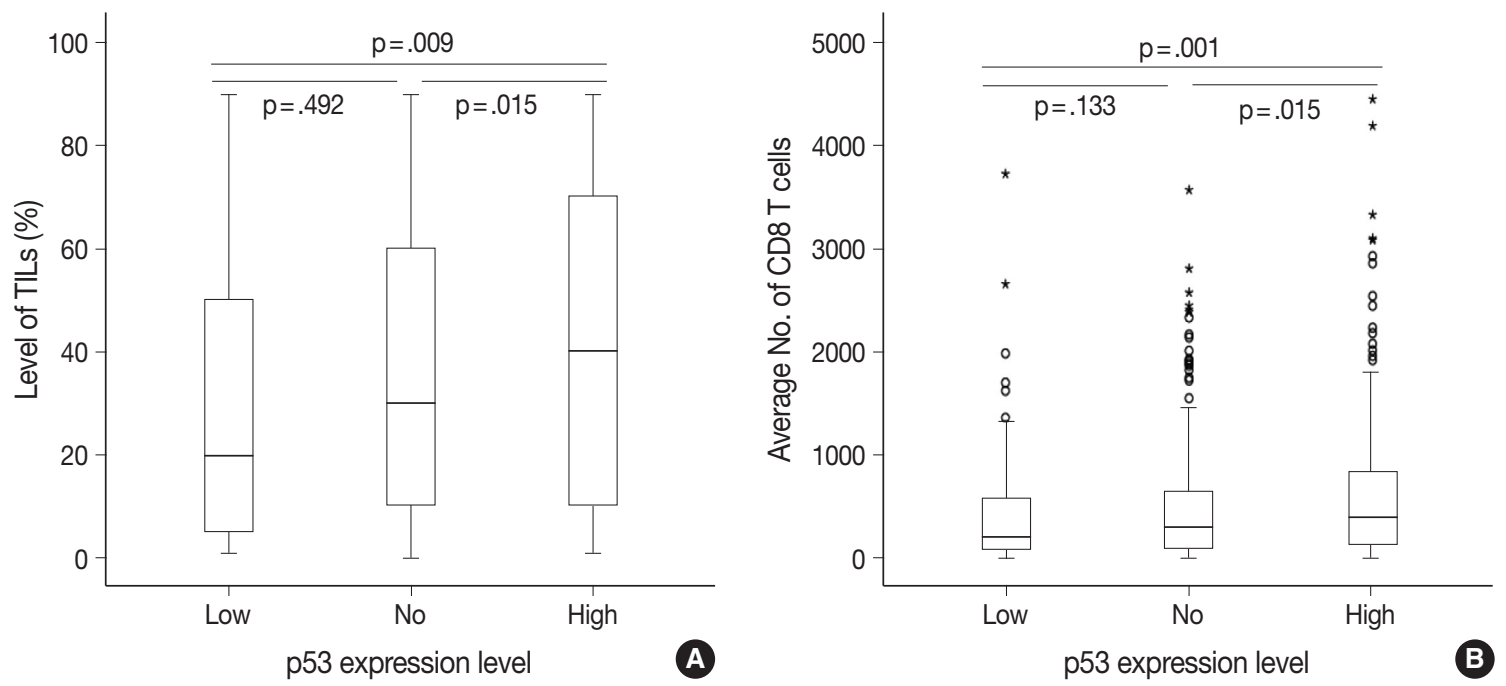

Fig. 3. Differences in the level of tumor-infiltrating lymphocytes (TILs) (A) and the average number of CD8 ${ }^{+} \mathrm{T}$ cells (B) among the three groups of triple-negative breast cancer patients categorized by p53 expression level.

group was significantly associated with higher TIL levels ( $\mathrm{p}=$ .032). Compared to the low p 53 expression group, the no $\mathrm{p} 53$ expression group was significantly associated with higher histologic grade $(\mathrm{p}=.011)$. The TIL levels of the no $\mathrm{p} 53$ expression group did not show significant differences from the TIL levels of the low $\mathrm{p} 53$ expression group $(\mathrm{p}=.524)$.

There were differences in the amounts of TILs (Fig. 3A) and the average number of $\mathrm{CD} 8^{+} \mathrm{T}$ cells (Fig. $3 \mathrm{~B}$ ) among the three groups of TNBC patients classified according to $\mathrm{p} 53$ expression level. The high $\mathrm{p} 53$ expression group had significantly higher amounts of TILs $(\mathrm{p}=.009$ and $\mathrm{p}=.015)$ and more CD8+ T cells $(\mathrm{p}=.001$ and $\mathrm{p}=.015)$ than the low and no expression groups, respectively. However, there was no significant difference between the no and low $\mathrm{p} 53$ expression groups in the amounts of stromal TILs $(\mathrm{p}=.492)$ and the number of CD8 ${ }^{+} \mathrm{T}$ cells $(\mathrm{p}=.133)$. 
Association between high histologic grade and endoplasmic reticulum stress-associated molecules

Since tumors with high histologic grades have an increased proliferation rate, which induces endoplasmic reticulum stress, we also analyzed the relationship between histologic grade and expression of endoplasmic reticulum stress-associated molecules. High histologic grade was significantly associated with two out of three endoplasmic reticulum stress-associated molecules ( $\mathrm{p}$ eIF2a, $\mathrm{p}=.036$ and XBP1, $\mathrm{p}<.001$, but not PERK, $\mathrm{p}=.928$ ) (Table 2).

\section{Prognostic significance of p53 expression in TNBC}

Univariate analysis was undertaken to elucidate the prognostic significance of $\mathrm{p} 53$ expression. There was no significant difference in disease-free $(\mathrm{p}=.406)$ or overall survival rates $(\mathrm{p}=.444)$ among the three groups of no, low, and high p53 expression (Fig. 4).

Table 2. Comparison of pathologic variables according to histologic grade

\begin{tabular}{crcr}
\hline \multirow{2}{*}{ Parameter } & \multicolumn{3}{c}{ Histologic grade } \\
\cline { 2 - 4 } & 1 and 2 & 3 & p-value \\
\hline p-elF2a & $92(58.2)$ & $246(48.4)$ & .036 \\
Low & $66(41.8)$ & $262(51.6)$ & \\
High & & & .928 \\
PERK & $85(53.5)$ & $277(76.5)$ & \\
Low & $74(46.5)$ & $237(46.1)$ & \\
High & & & $<.001$ \\
XBP1 & $109(69.4)$ & $263(51.7)$ & \\
Low & $48(30.6)$ & $246(48.3)$ & \\
High & &
\end{tabular}

Values are presented as number (\%).

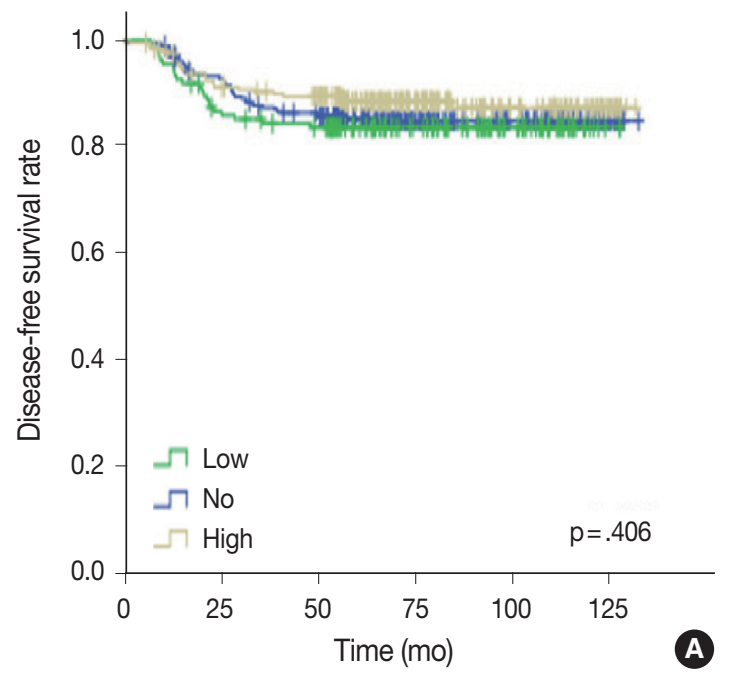

\section{Prognostic significance of TILs among groups based on p53 expression in TNBC}

Since the level of TILs in TNBC patients has been recognized as a significant prognostic factor, ${ }^{24}$ the effect of TILs in each p 53 expression group was examined. In the high $\mathrm{p} 53$ expression group, the higher the TIL level, the better the disease-free ( $\mathrm{p}=.006)$ and overall survival rates $(\mathrm{p}=.005)$ (Fig. 5). Disease-free $(\mathrm{p}=.001)$ and overall survival curves $(\mathrm{p}=.001)$ were more significantly separated when the TILs were divided by $10 \%$. In the no p53 expression group, the higher the TIL level, the higher the diseasefree $(\mathrm{p}=.044)$ and overall survival rates $(\mathrm{p}=.046)$. Conversely, in the low $\mathrm{p} 53$ expression group, disease-free $(\mathrm{p}=.092)$ and overall survival rates $(\mathrm{p}=.108)$ were not significantly different, according to the TIL levels.

\section{DISCUSSION}

Our previous studies demonstrated that TILs are highly associated with interferon- and endoplasmic reticulum stress-associated molecules. ${ }^{5,8,25}$ Based on these results, we hypothesized that high p53 expression, which is linked with a high histologic grade (frequent mitosis, large cell and nuclear size, and poor differentiation), might induce endoplasmic reticulum stress and subsequent interferon signaling pathway activation, as well as the influx of TILs. Moreover, high p 53 expression, which is related to necrosis, might induce the release of damage-associated molecules, an immune response, and the influx of TILs. The present research analyzed the clinical and pathological significance of p53 expression in TNBC. In comparison to low p53 expression, high $\mathrm{p} 53$ expression was shown to be associated with high his-

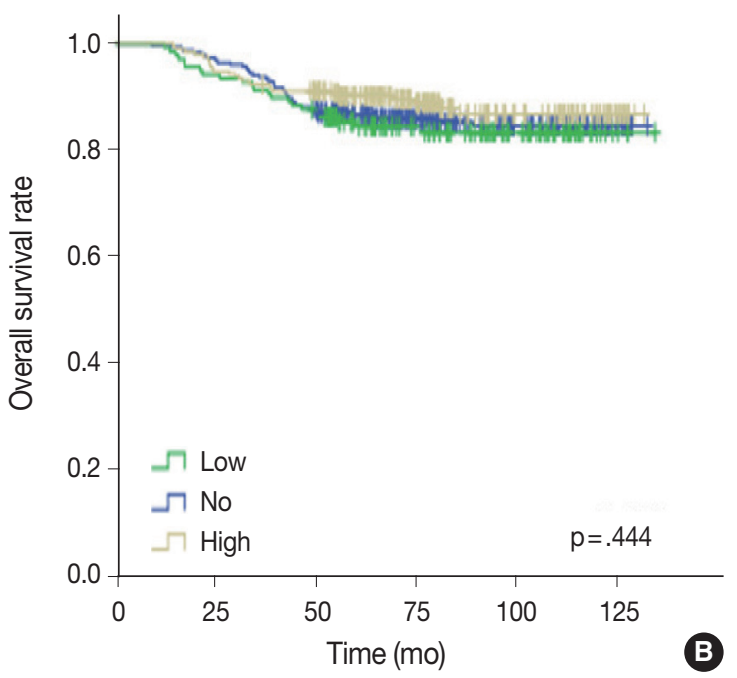

Fig. 4. (A, B) Kaplan-Meier survival analyses of triple-negative breast cancer patients categorized by p53 expression levels. 

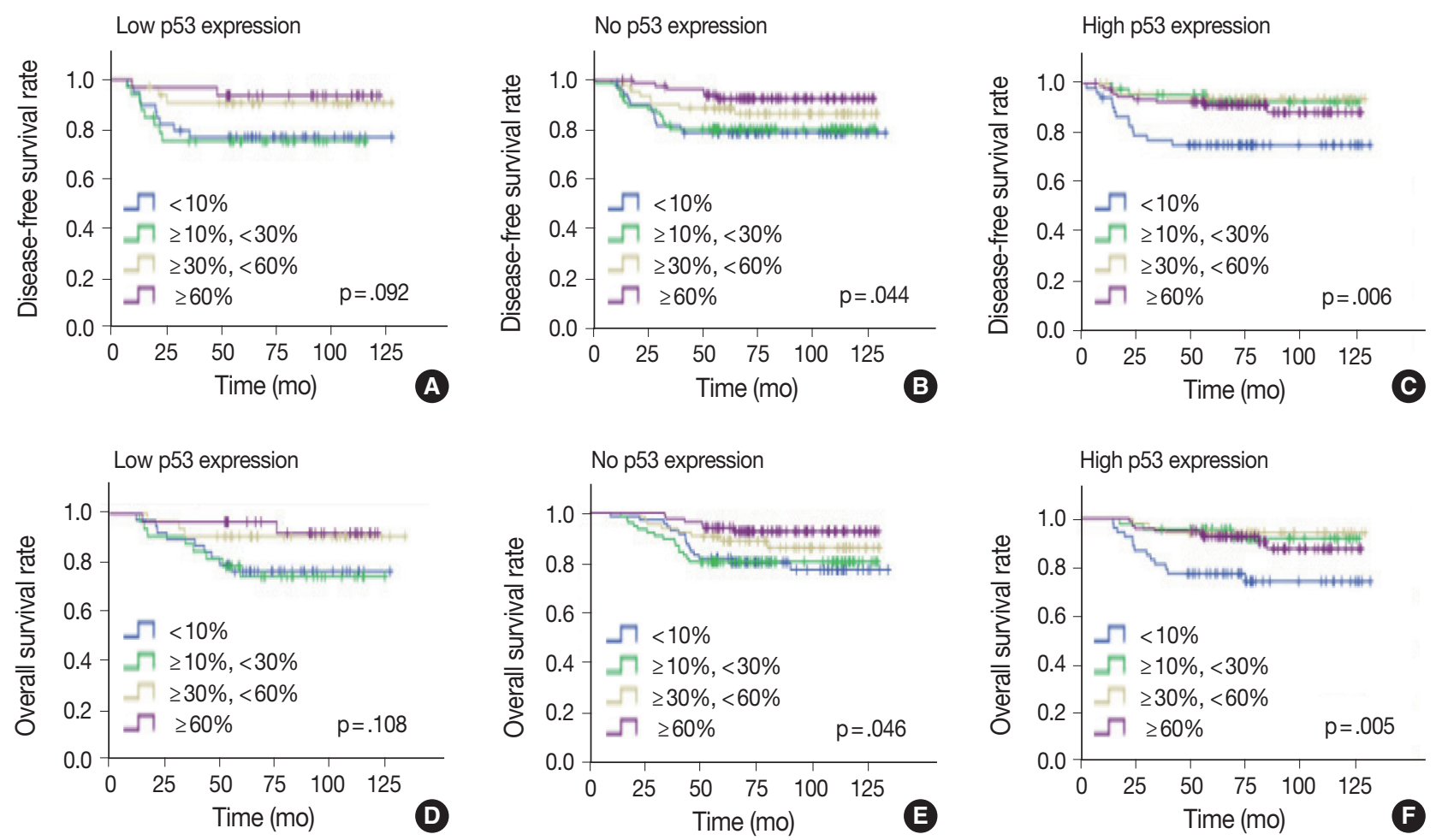

Fig. 5. Kaplan-Meier survival analyses of triple-negative breast cancer patients according to tumor-infiltrating lymphocytes levels in low (A, $\mathrm{D})$, no (B, E), and high (C, F) p53 expression groups.

tologic grade, necrosis in the invasive area, and more TILs, supporting the study hypothesis.

Darb-Esfahani et al. ${ }^{26}$ revealed a significant association between p53 protein expression characterized by immunohistochemical staining intensity ("wild-type pattern", tumor cell nuclei stained as variable and weak intensity; "overexpression", $\geq 60 \%$ of tumor cell nuclei stained as uniformly strong or moderate intensity; "loss," tumor cell nuclei stained completely negative) and the TP53 mutation, with $80 \%$ of the $\mathrm{p} 53$ overexpression group possessing the missense mutation. Also, in TNBC, missense TP53 mutations were significantly linked with higher levels of stromal TILs $(\mathrm{p}=.028)$ and $C D 8 A$ gene expression $(\mathrm{p}=.020)$, and tended to be associated with a better survival $(\mathrm{p}=.093)$ compared to all other types of mutations. ${ }^{26}$

TNBC has a higher TIL level than other breast cancer subtypes. However, the level of TIL varies within TNBC. TP53 mutations occur more frequently in TNBC (80\%) than other breast cancer subtypes. ${ }^{2,10}$ Of the TP 53 mutations, missense mutations produce a new mutant protein that can be presented on the cell surface via major histocompatibility complex (neoantigen), triggering the immune system and leading to a TIL response. This hypothesis is supported by our finding that the TIL levels of p53 overexpression group (probably due to missense muta- tion) were significantly higher than the TIL levels in the p53 low expression group (wild-type TP53) ( $\mathrm{p}=.009$ ). In contrast, null mutations of the TP53 gene simply do not produce normal levels of $\mathrm{p} 53$ protein, and the $\mathrm{p} 53$ function is lost. Since it does not make a new mutant protein to act as a neoantigen, it does not trigger the immune response, resulting in unchanged TIL levels. This hypothesis is supported by our finding that the no p53 expression group (probably due to null mutation) did not show a significant difference in TIL levels compared to those of the low $\mathrm{p} 53$ expression group $(\mathrm{p}=.524)$, while TIL levels in the no 553 expression group showed a significant difference compared to TIL levels in the high $\mathrm{p} 53$ expression group ( $\mathrm{p}=.032$ ). Although TNBC has a high mutation burden and other gene mutations can enhance immunogenicity, our findings suggest an important role of TP53 mutation in the immunogenicity of TNBC.

In TNBC patients, a high TIL level is associated with good prognosis. ${ }^{24}$ In our study, p 53 overexpression was related to TIL levels, but not with prognosis. Although there have been many attempts to correlate TP53 mutation status and clinical outcomes, such as overall survival rates in TNBC patients, conflicting findings have often emerged, with either poor survival in TNBC patients with TP53 mutation ${ }^{27-30}$ or no significance. ${ }^{31,32}$ This is 
probably due to the beneficial effects of mutant p 53 gain-offunction for tumorigenesis that offsets the favorable prognostic impacts of high TIL levels.

Mutant $\mathrm{p} 53$ proteins have been regarded as attractive targets for cancer therapy. Most of the strategies developed to target mutant $\mathrm{p} 53$ proteins involve restoration to wild-type $\mathrm{p} 53$ activity and depletion of the mutant $\mathrm{p} 53$ protein. Small molecules, such as 2,2-bis(hydroxymethyl)-quinuclidin-3-one or zinc metallochaperone-1, are representative restoration methods to wild-type p53 activity. Among exemplary methods for depletion of the mutant p 53 protein, geldanamycin, 17AAG, and ganetespib are inhibitors of heat shock protein 90, which, in turn, inhibits the degradation of mutant $\mathrm{p} 53$ protein mediated by carboxyl terminus of heat shock cognate protein 70-interacting protein and murine double minute 2 (MDM2). ${ }^{16,33}$

In the current study, overall and disease-free survival rates were much better when the level of TILs was $\geq 10 \%$ compared with $<10 \%$ in the high $\mathrm{p} 53$ expression group, suggesting that the TP53 missense mutation is associated with TIL influx and some TILs can recognize mutant $\mathrm{p} 53$. Therefore, in the high $\mathrm{p} 53$ expression groups with TIL levels $<10 \%$, the identification of $\mathrm{T}$ cells with $\mathrm{T}$ cell receptors (TCRs) reactive to mutant $\mathrm{p} 53$ and development of engineered $\mathrm{T}$ cell adoptive immunotherapy targeting mutant p 53 may lead to successful immunotherapy. For instance, Lheureux et al..$^{33}$ found immunogenic TP53 "hotspot" mutations as well as T cells with mutant $\mathrm{p} 53$-reactive TCRs in seven ovarian cancer patients. Therefore, in TNBCs with TP53 mutations, genetic transfer of the TP53 mutation-specific TCRs into autologous lymphocytes could be used to generate cells for use in cancer adoptive cell transfer immunotherapy.

The present study demonstrated close associations between the expression of $\mathrm{p} 53$ and the molecules associated with endoplasmic reticulum stress and TIL influx. Further studies targeting mutant $\mathrm{p} 53$ could facilitate the development of efficient immunotherapeutic agents.

\section{ORCID}

Miseon Lee: https://orcid.org/0000-0002-6385-7621

In Ah Park: https://orcid.org/0000-0002-9542-9461

Sun-Hee Heo: https://orcid.org/0000-0002-3043-4139

Young-Ae Kim: https://orcid.org/0000-0002-5454-7816

Gyungyub Gong: https://orcid.org/0000-0001-5743-0712

Hee Jin Lee: https://orcid.org/0000-0002-4963-6603

\section{Author Contributions}

Conceptualization: HJL.

Data curation: IAP, ML, HJL.

Formal analysis: IAP, ML.

Funding acquisition: IAP, GG, HJL.

Investigation: $\mathrm{HJL}$.

Methodology: HJL.

Project administration: ML.

Resources: GG, HJL.

Supervision: HJL.

Validation: HJL.

Visualization: IAP, ML.

Writing—original draft: IAP, ML.

Writing—review \& editing: SHH, YAK, GG, HJL.

\section{Conflicts of Interest}

The authors declare that they have no potential conflicts of interest.

\section{Acknowledgments}

This study was funded by the Basic Science Research Program through the National Research Foundation of Korea (NRF) supported by the Ministry of Science, ICT \& Future Planning (grant number 2017R1D1A1B03035491).

\section{REFERENCES}

1. Yamashita N, Kondo M, Zhao S, et al. Picrasidine G decreases viability of MDA-MB 468 EGFR-overexpressing triple-negative breast cancer cells through inhibition of EGFR/STAT3 signaling pathway. Bioorg Med Chem Lett 2017; 27: 2608-12.

2. Yadav BS, Chanana $\mathrm{P}$, Jhamb S. Biomarkers in triple negative breast cancer: a review. World J Clin Oncol 2015; 6: 252-63.

3. van Rooijen JM, Stutvoet TS, Schroder CP, de Vries EG. Immunotherapeutic options on the horizon in breast cancer treatment. Pharmacol Ther 2015; 156: 90-101.

4. Kim JY, Heo SH, Song IH, et al. Activation of the PERK-eIF2alpha pathway is associated with tumor-infiltrating lymphocytes in HER2-positive breast cancer. Anticancer Res 2016; 36: 2705-11.

5. Kim YA, Lee HJ, Heo SH, et al. MxA expression is associated with tumor-infiltrating lymphocytes and is a prognostic factor in triplenegative breast cancer. Breast Cancer Res Treat 2016; 156: 597-606.

6. Lee HJ, Park IA, Song IH, et al. Tertiary lymphoid structures: prognostic significance and relationship with tumour-infiltrating lymphocytes in triple-negative breast cancer. J Clin Pathol 2016; 69: $422-30$. 
7. Lee HJ, Song IH, Park IA, et al. Differential expression of major histocompatibility complex class I in subtypes of breast cancer is associated with estrogen receptor and interferon signaling. Oncotarget 2016; 7: 30119-32.

8. Park IA, Heo SH, Song IH, et al. Endoplasmic reticulum stress induces secretion of high-mobility group proteins and is associated with tumor-infiltrating lymphocytes in triple-negative breast cancer. Oncotarget 2016; 7: 59957-64.

9. Chen X, Iliopoulos D, Zhang Q, et al. XBP1 promotes triple-negative breast cancer by controlling the HIFlalpha pathway. Nature 2014; 508: 103-7.

10. Han CC, Wan FS. New insights into the role of endoplasmic reticulum stress in breast cancer metastasis. J Breast Cancer 2018; 21: 35462.

11. Oros Klein K, Oualkacha K, Lafond MH, Bhatnagar S, Tonin PN, Greenwood CM. Gene coexpression analyses differentiate networks associated with diverse cancers harboring TP53 missense or null mutations. Front Genet 2016; 7: 137.

12. Kandioler-Eckersberger D, Ludwig C, Rudas M, et al. TP53 mutation and p53 overexpression for prediction of response to neoadjuvant treatment in breast cancer patients. Clin Cancer Res 2000; 6: 50-6.

13. Brosh R, Rotter V. When mutants gain new powers: news from the mutant p53 field. Nat Rev Cancer 2009; 9: 701-13.

14. Freed-Pastor WA, Prives C. Mutant p53: one name, many proteins. Genes Dev 2012; 26: 1268-86.

15. Muller PA, Vousden KH. p53 mutations in cancer. Nat Cell Biol 2013; 15: 2-8.

16. Yue X, Zhao Y, Xu Y, Zheng M, Feng Z, Hu W. Mutant p53 in cancer: accumulation, gain-of-function, and therapy. J Mol Biol 2017; 429: 1595-606.

17. Alsner J, Yilmaz M, Guldberg P, Hansen LL, Overgaard J. Heterogeneity in the clinical phenotype of TP53 mutations in breast cancer patients. Clin Cancer Res 2000; 6: 3923-31.

18. Jin MS, Park IA, Kim JY, et al. New insight on the biological role of p53 protein as a tumor suppressor: re-evaluation of its clinical significance in triple-negative breast cancer. Tumour Biol 2016; 37: 11017-24.

19. Cancer Genome Atlas Network. Comprehensive molecular portraits of human breast tumours. Nature 2012; 490: 61-70.

20. Duffy MJ, Synnott NC, McGowan PM, Crown J, O'Connor D, Gallagher WM. p53 as a target for the treatment of cancer. Cancer Treat Rev 2014; 40: 1153-60.

21. Duffy MJ, Synnott NC, Crown J. Mutant p53 as a target for cancer treatment. Eur J Cancer 2017; 83: 258-65.
22. Kojima YA, Wang X, Sun H, Compton F, Covinsky M, Zhang S. Reproducible evaluation of tumor-infiltrating lymphocytes (TILs) using the recommendations of International TILs Working Group 2014. Ann Diagn Pathol 2018; 35: 77-9.

23. Park IA, Hwang SH, Song IH, et al. Expression of the MHC class II in triple-negative breast cancer is associated with tumor-infiltrating lymphocytes and interferon signaling. PLoS One 2017; 12: e0182786.

24. Adams S, Goldstein LJ, Sparano JA, Demaria S, Badve SS. Tumor infiltrating lymphocytes (TILs) improve prognosis in patients with triple negative breast cancer (TNBC). Oncoimmunology 2015; 4: e985930.

25. Lee HJ, Kim A, Song IH, et al. Cytoplasmic expression of high mobility group B1 (HMGB1) is associated with tumor-infiltrating lymphocytes (TILs) in breast cancer. Pathol Int 2016; 66: 202-9.

26. Darb-Esfahani S, Denkert C, Stenzinger A, et al. Role of TP53 mutations in triple negative and HER2-positive breast cancer treated with neoadjuvant anthracycline/taxane-based chemotherapy. Oncotarget 2016; 7: 67686-98.

27. Maeda T, Nakanishi Y, Hirotani Y, et al. Immunohistochemical co-expression status of cytokeratin $5 / 6$, androgen receptor, and p53 as prognostic factors of adjuvant chemotherapy for triple negative breast cancer. Med Mol Morphol 2016; 49: 11-21.

28. Wu M, Wei W, Xiao X, et al. Expression of SIRT1 is associated with lymph node metastasis and poor prognosis in both operable triplenegative and non-triple-negative breast cancer. Med Oncol 2012; 29: 3240-9.

29. Zhang J, Wang Y, Yin Q, Zhang W, Zhang T, Niu Y. An associated classification of triple negative breast cancer: the risk of relapse and the response to chemotherapy. Int J Clin Exp Pathol 2013; 6: 1380-91.

30. Biganzoli E, Coradini D, Ambrogi F, et al. p53 status identifies two subgroups of triple-negative breast cancers with distinct biological features. Jpn J Clin Oncol 2011; 41: 172-9.

31. Kashiwagi S, Yashiro M, Takashima T, et al. Advantages of adjuvant chemotherapy for patients with triple-negative breast cancer at Stage II: usefulness of prognostic markers E-cadherin and Ki67. Breast Cancer Res 2011; 13: R122.

32. Wang J, Zhang C, Chen K, et al. ERbeta1 inversely correlates with PTEN/PI3K/AKT pathway and predicts a favorable prognosis in triple-negative breast cancer. Breast Cancer Res Treat 2015; 152: 255-69.

33. Lheureux S, Denoyelle C, Ohashi PS, De Bono JS, Mottaghy FM. Molecularly targeted therapies in cancer: a guide for the nuclear medicine physician. Eur J Nucl Med Mol Imaging 2017; 44(Suppl 1): $41-54$. 